\title{
El rol de la hipertensión pulmonar en la displasia broncopulmonar
}

\author{
The role of pulmonary hypertension on bronchopulmonary dysplasia
}

\author{
Valenzuela D. ${ }^{\mathrm{a}}$, Moya F. ${ }^{\mathrm{a}}$, Luco M. ${ }^{\mathrm{b}}$, Tapia JL. ${ }^{\mathrm{b}}$ \\ aFacultad de Medicina, Pontificia Universidad Católica de Chile \\ ${ }^{b}$ Departamento Neonatología, Pontificia Universidad Católica de Chile
}

Recibido el 31 de julio de 2017; aceptado el 26 de septiembre de 2017

\begin{abstract}
Resumen
Hace 50 años Northway describió la Displasia Broncopulmonar (DBP), en nacidos de pretérmino expuestos a ventilación mecánica. Desde entonces, ha aumentado la sobrevida de ellos; sin embargo, ha aparecido una "nueva DBP" y la incidencia de esta no ha disminuido. Una de las características de esta patología es la remodelación vascular anómala, que en su expresión más severa se conoce como Hipertensión Pulmonar (HP); con una incidencia de 17\%, que es proporcional a la severidad de la DBP (33\% en DBP severa); y como un factor de mortalidad (hasta un $48 \%$ mortalidad a 2 años con HP por DBP). Debido a esto resulta importante conocer los métodos diagnósticos y alternativas terapéuticas, tema que se discute en esta revisión. Considerando la alta mortalidad de la asociación HP-DBP, adquiere importancia una estrategia de tamizaje en la población de riesgo. El gold standard para el diagnóstico de HP es el cateterismo cardíaco, sin embargo, el ecocardiograma transtorácico es una herramienta útil para el tamizaje y diagnóstico de HP en pacientes displásicos, con mediciones cuantitativas y cambios cualitativos en la evaluación diagnóstica. A nivel sanguíneo el péptido natriurético tipo B (BNP), ha mostrado ser útil en el seguimiento; en cuanto a imágenes, la tomografía computarizada se utiliza en casos severos. En cuanto a las terapias, se han propuesto el óxido nítrico inhalado como vasodilatador pulmonar, los inhibidores de la fosfodiesterasas -sildenafil-, los antagonistas de la endotelina -bosentán- y los análogos de prostaciclinas -iloprost-. Aún no se cuenta con evidencia de alta calidad para su uso, dosis y duración del tratamiento, pero hay variadas experiencias clínicas. Además, es relevante el cuidado interdisciplinario, destacando optimizar la nutrición. El desafío es lograr una prevención efectiva de la DBP y de sus complicaciones. Un protocolo de tamizaje de HP debe asociarse a una estratificación de riesgo y directrices de tratamiento.
\end{abstract}

Palabras clave:

Displasia

broncopulmonar, Hipertensión pulmonar, enfermedad pulmonar crónica, recién nacido prematuro 


\section{Abstract}

50 years ago, Northway described Broncopulmonary Dysplasia (BPD) in preterm infants exposed to mechanical ventilation. Since then, their survival has increased, nevertheless a "new BPD" has appeared and its incidence has not diminished. One of the characteristics of this pathology is the the abnormal vascular remodeling, which in its most severe expression is known as Pulmonary Hypertension $(\mathrm{PH})$; with an incidence of $17 \%$ in patients with $\mathrm{BPD}$, which is proportional to the severity of the disease (33\% in severe BPD), and as mortality factor (up to 48\% 2-year mortality in PH-BPD). Thereby, it is important to know the diagnostic methods and therapeutic alternatives, topics discussed in this review. Considering the high mortality in BPD associated $\mathrm{PH}$, screening strategies in at risk population become important. The gold standard is cardiac catheterization; however, transthorathic echocardiography is a useful tool for the screening and diagnosis of $\mathrm{PH}$ in displasic patients, using cuantitive measures and cualitative changes in the evaluation. Seric type-B natriuretic peptide has shown to be useful for follow-up; regarding images, CT scan is used in severe cases. In terms of therapy; inhaled Nitric Oxide as a pulmonary vasodilator, phosphodiesterase inhibitors -sildenafil-, endotelin antagonists -bosentan-, and prostacyclin analogues -iloprost-, have been proposed. Their use, dosis and treatment lenght still lack support of high quality evidence, but diverse clinical experiences have been described. Interdisciplinary care is also important, highlighting to optimize nutrition. Therefore, the challenge is to effectively prevent BPD and its complications. A PH screening protocol should be associated with risk stratification and treatment guidelines.

\section{Keywords:}

Bronchopulmonary dysplasia, pulmonary dysplasia, chronic lung disease, preterm newborns

\section{Introducción}

Se cumplen 50 años desde que Northway y sus colegas describieron por primera vez la enfermedad pulmonar crónica que se presenta en recién nacidos (RN) de pretérmino sobrevivientes a una Enfermedad de Membrana Hialina expuestos a ventilación mecánica prolongada y agresiva con altas concentraciones de oxígeno y la denominaron "Displasia Broncopulmonar" (DBP) $)^{1}$.

Actualmente la definición derivada del consenso del Instituto Nacional de Salud de EEUU es la más aceptada. En ella se considera los días de oxigenoterapia y el uso de éste a las 36 semanas de edad corregida o 56 días de vida (según sea la edad gestacional al nacer) para identificar la severidad de la patología.

En las últimas décadas hemos sido testigos como la DBP continúa siendo la secuela crónica más común en los prematuros de muy bajo peso, y que su incidencia, lejos de disminuir, se mantiene o incluso en algunas series aumenta ${ }^{2,3}$.

En Chile, calculamos que se presentan aproximadamente 350 nuevos casos de DBP cada año.

En la Unidad de Neonatología de la Universidad Católica, entre los años 2005-2014, la incidencia de DBP en los menores de $1.500 \mathrm{~g}$ fue de un 23,9\%, siendo moderada o severa en el 13,3\%. En la Red sudamericana Neocosur, estas cifras son de aproximadamente un $25 \%$ y $17,5 \%$ respectivamente ${ }^{4}$.

Esta falla en disminuir la DBP se debe al aumento en la sobrevida de la población de prematuros extremos, secundaria al mayor uso de corticoides antenatales, de surfactante exógeno; sumado al mejor manejo ventilatorio y nutricional de nuestros pacientes. Esto, no sólo ha llevado a que la incidencia de la DBP se mantenga alta, sino que también a que la fisiopatología y la expresión de la DBP haya cambiado y se describa lo que se ha llamado la "nueva DBP".

Investigaciones recientes han puesto en evidencia el rol central que tendrían las alteraciones en el desarrollo de la vasculatura pulmonar en la expresión de esta nueva DBP, y como a su vez la Hipertensión Pulmonar (HP) sería una expresión de esta enfermedad vascular y que incidiría directamente en el pronóstico de los niños portadores de esta nueva DBP. En algunos estudios se ha demostrado que la presencia de HP conlleva un aumento de la mortalidad en los pacientes portadores de DBP, llegando ésta hasta un $48 \%$ a los 2 años en pacientes diagnosticados con esta asociación ${ }^{5}$.

En un metaanálisis publicado este año por AlGhanem et al, se encontró una incidencia de HP en pacientes con DBP de $17 \%$, pudiéndose identificar un $4 \%$ en DBP leve, versus un $33 \%$ en DBP severa, demostrando una clara asociación entre la presencia de HP y la severidad de la DBP. Además, en este trabajo se reportó una mayor morbimortalidad en los pacientes con HP asociada a DBP en comparación a los pacientes que sólo presentaban DBP. Las otras variables estudiadas (duración inicial de hospitalización, duración de oxigenoterapia, requerimientos de oxígeno domiciliario) no mostraron diferencias entre la población de pacientes displásicos con HP versus sin HP, y no se encontraron estudios que reportaran resultados a largo plazo 6 .

El objetivo de esta actualización es recopilar la evi- 
dencia que asocian la HP y la DBP, y a la vez sugerir algunos lineamientos de prevención, evaluación y tratamiento de esta asociación.

\section{Patogénesis}

La etiopatogenia multifactorial de la DBP descrita por Northway se mantiene aún vigente. Por lo que siguen teniendo un rol importante en el desarrollo de DBP la prematuridad, el síndrome de dificultad respiratoria, y la injuria producida por ventilación mecánica y el oxígeno en altas concentraciones ${ }^{1}$.

Debido al mayor conocimiento y la evolución de esta entidad en el tiempo, se han identificado nuevos factores, incluyendo algunos prenatales: restricción del crecimiento intrauterino, inflamación y/o infección, postnatales: administración excesiva de líquidos, edema pulmonar, persistencia del ductus arterioso, déficit nutricional, y otros intrínsecos del recién nacido: inmadurez de los sistemas antioxidantes, insuficiencia adrenal precoz, y predisposición genética.

Esta compleja interacción entre factores maternos, genéticos y ambientales, contribuyen a alteraciones en la expresión normal de factor de crecimiento y en sus vías de señalización, conduciendo a un deterioro del crecimiento, la estructura y función del desarrollo de la circulación pulmonar en el prematuro (figura 1).

El crecimiento anormal de la circulación pulmonar en DBP se caracteriza por una disminución de la ramificación vascular, un patrón alterado de la distribución vascular en el intersticio pulmonar y anastomosis venosas intrapulmonares persistentes, lo que en con- junto contribuyen a los síntomas de la DBP. La injuria endotelial causada por estresores ambientales induce a la proliferación de células de músculo liso en las pequeñas arterias pulmonares; se produce maduración precoz de células mesenquimales inmaduras a células maduras de músculo liso e incorporación de fibroblastos y miofibroblastos en la pared vascular. Por lo tanto, no sólo nos encontramos frente a una alteración de daño alveolar con simplificación de su septación, sino que también dentro de la fisiopatología de la DBP existe una enfermedad vascular pulmonar, donde el desarrollo anormal de la microvasculatura junto al remodelamiento de la vasculatura pulmonar resultan en una disminución del área de sección transversal y con ello un aumento de la resistencia vascular pulmonar. Estas anomalías persistentes del crecimiento vascular pulmonar contribuyen al desarrollo de enfermedad vascular pulmonar, la cual se vuelve cada vez más sintomática, pudiendo llegar a su expresión más severa en la $\mathrm{HP}^{7}$.

\section{Evaluación}

Estudios recientes sugieren que la presencia de HP se asocia con mayor morbimortalidad en pacientes con DBP, evidenciándose una tasa de mortalidad de 33\%$48 \%$ a los 2 años del diagnóstico de HP, y que esta es más frecuente mientras más severa sea la $\mathrm{DBP}^{5}$. Debido a esto, la realización de un tamizaje de rutina podría ser útil para identificar esta patología en aquella población de alto riesgo.

A pesar de que la cateterización cardiaca es ac-

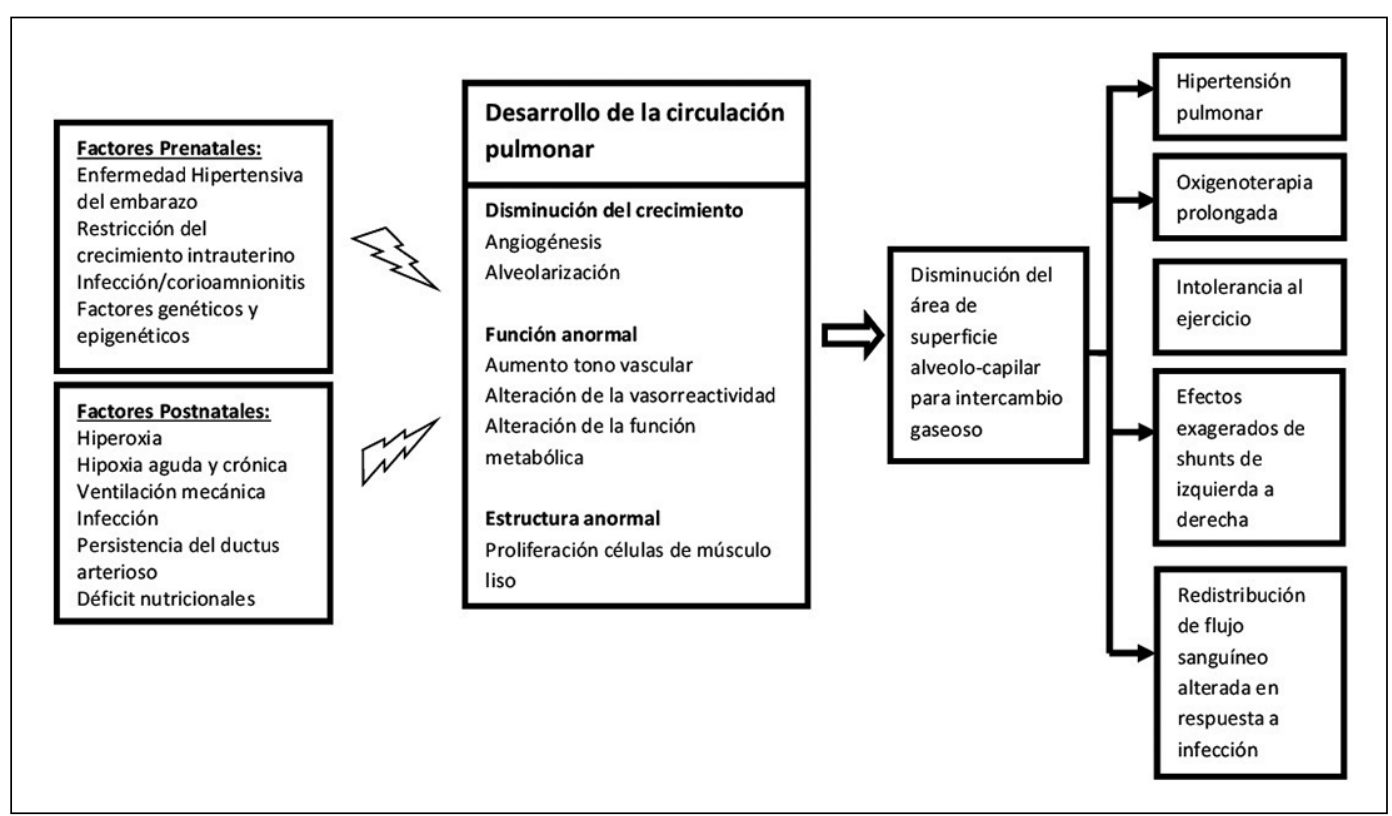

Figura 1. Patogenia de HP en DBP y manifestación clínica. Adaptado de Mourani et al $2009^{26}$ 
tualmente el Gold estándar para el diagnóstico de $\mathrm{HP}$, definiéndose como presión de arteria pulmonar $>25 \mathrm{mmHg}$; la evaluación con Ecocardiografía transtorácica, al ser un procedimiento no invasivo, se ha convertido en una herramienta muy útil para el tamizaje y diagnóstico de HP. Una medida comúnmente usada para el diagnóstico de HP es la estimación de presión sistólica de ventrículo derecho derivada de la velocidad máxima de regurgitación tricuspídea, según fórmula de Bernoulli ${ }^{7}$. En general la HP es considerada como leve cuando la presión sistólica del ventrículo derecho no alcanza la mitad de la presión sistólica sistémica; moderada, cuando ésta se encuentra entre la mitad y $2 / 3$ la sistémica y severa cuando se sobrepasa este último límite. Dentro de las características cualitativas observadas se encuentran la hipertrofia de ventrículo derecho, aplanamiento del septum interventricular, dilatación de aurícula derecha y dilatación de ventrículo derecho ${ }^{8,9,10}$ (figura 2).

En un estudio reciente, Carlton et al, determinó la concordancia inter-operadores en evaluaciones ecocardiográficas para HP realizada a las 36 semanas de edad gestacional corregida en recién nacidos menores de 1.250 g (17,8\% y $15,6 \%$, Kappa score ajustado de $0,8)$, sugiriendo que el establecimiento de un protocolo de evaluación ecocardiográfica podría mejorar la identificación de enfermedad vascular pulmonar en RN prematuros?.

A pesar de su utilidad de screening diagnóstico, la ecocardiografía aún presenta limitaciones en comparación al cateterismo. Mourani et al 2008 realizó un estudio retrospectivo donde se evaluaron 25 pacientes a los cuales se les realizó ecocardiografía transtorácica con posterior cateterización cardiaca para la evaluación de HP. La estimación de la presión sistólica de arteria pulmonar medida por ecocardiografía diagnosticó correctamente la presencia o ausencia de HP en un 79\% de los pacientes, pero determinó severidad solo en un 47\%. Además, la ecocardiografía falló en diagnosticar HP en
$11 \%$ de los pacientes (falsos negativos), e inadecuadamente diagnosticó HP en un $11 \%$ (falsos positivos) ${ }^{11}$. Sin embargo la no concomitancia de la realización de las evaluaciones, junto a la diferencia de condiciones en que éstas fueron realizadas (anestesia + ventilación mecánica $v s$ niño despierto) puede haber incidido en estos resultados. Por otro lado, la cateterización cardíaca permite también la detección de lesiones como estenosis de vena pulmonar y colaterales que pueden presentarse en DBP, pero que no son fáciles de diagnosticar mediante ecografía. Es por esto que las guías de Hipertensión Pulmonar publicadas por la American Heart Association recomienda considerar el cateterismo cardiaco en algunos casos específicos como: 1) signos persistentes de enfermedad cardiorrespiratoria severa o deterioro clínico no explicado directamente por otra evaluación diagnóstica; 2) sospecha de tener HP significativa a pesar de manejo óptimo; 3) consideración de terapia vasodilatadora crónica; 4) presentación de edema pulmonar recurrente inexplicado ${ }^{7,12}$.

El objetivo del cateterismo cardiaco sería evaluar con mayor precisión la severidad de la HP, diagnosticar y medir el rol de cortocircuitos intra o extra-cardiacos, diagnosticar la eventual presencia de estenosis de las venas pulmonares y por último medir la reactividad vascular pulmonar en relación a terapias específicas (figura 3).

\section{Tomografía computada (TC)}

El estudio con tomografía computada de alta resolución en pacientes con DBP podría ser útil en determinar si existen anormalidades cardiovasculares asociadas y evaluar el compromiso de parénquima y vasculatura pulmonar. Del Cerro el al evaluó 29 pacientes con DBP, en los cuales se le realizó TC a 21 pacientes y cateterización cardiaca a 14 , evidenciándose anomalías cardiovasculares en $2 / 3$ de los pacientes, incluyendo colaterales aortopulmonares, estenosis de vena pulmonar, ductus arterioso persistente y defecto

Figura 2. Ecocardiograma. A: Eje corto de paciente sin signos de HP, nótese septum interventricular convexo al ventrículo derecho (VD). B: Eje corto de paciente con HP severa, nótese septum interventricular cóncavo al VD.

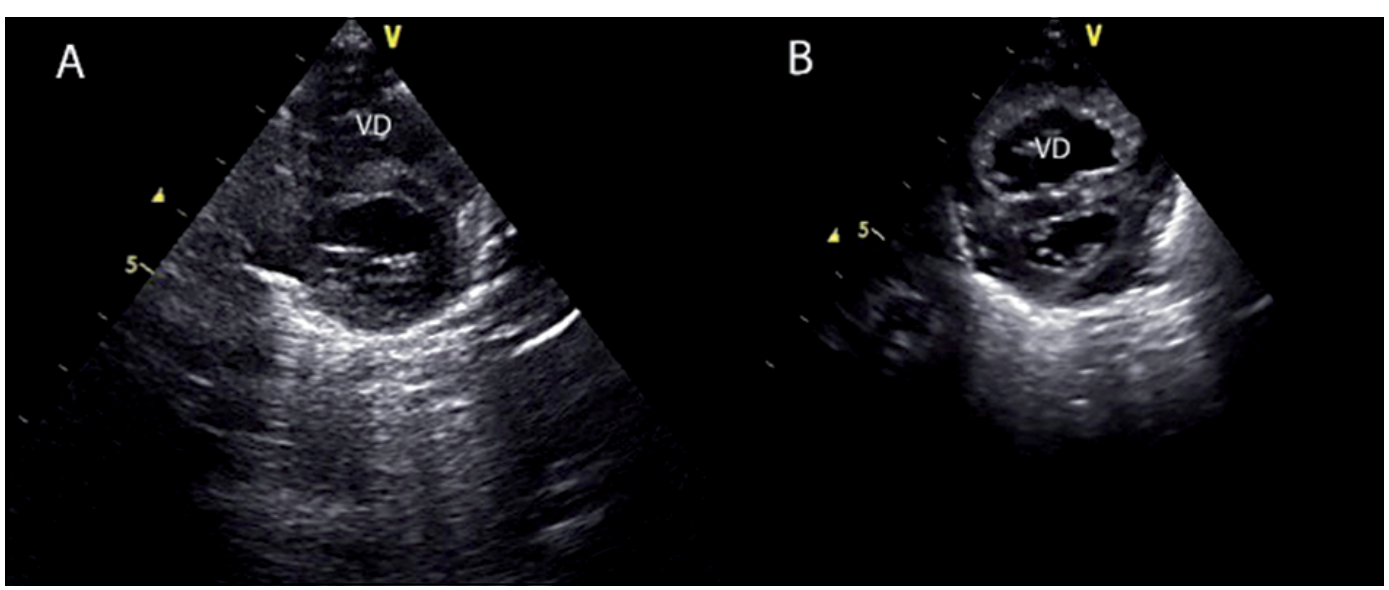




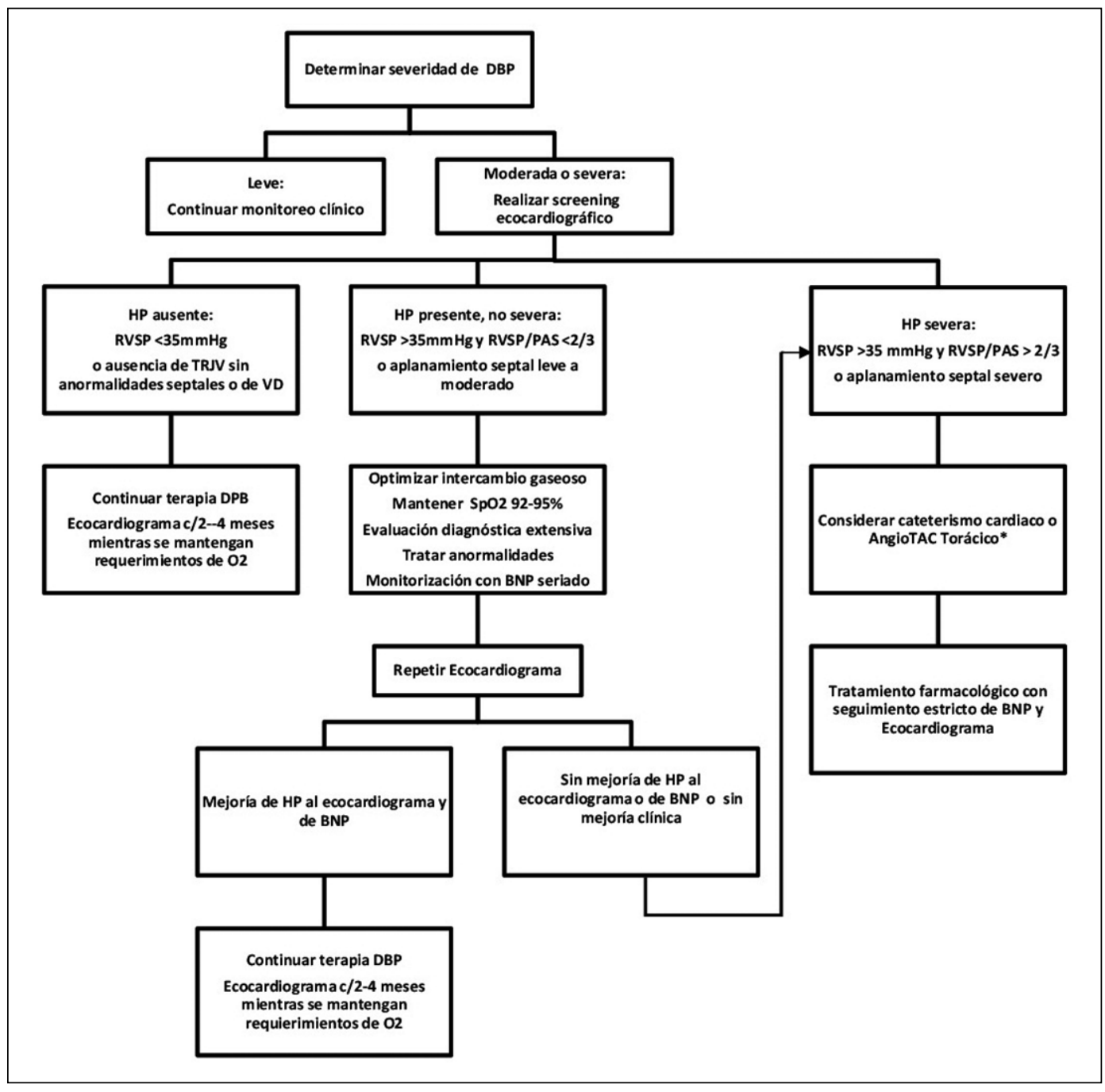

Figura 3. Flujograma de evaluación y manejo de HP en DBP. RVSP, presión sistólica de ventrículo derecho; TRJV, velocidad máxima de regurgitación tricuspídea; PAS, presión arterial sistólica sistémica; BNP, péptido natriurético tipo B. *Para evaluar eventuales comorbilidades como estenosis de venas pulmonares $y$ cortocircuitos intra y extracardíacos. (Modificado de Krishnan et al $2015^{28}$ y del Cerro et al $\left.2014^{10}\right)$.

de septum atrial, algunas de estas difíciles de evaluar o pesquisar por medio de la ecocardiografía. Estos autores recomiendan su uso en todo RN que persista con HP moderada o severa a los 2 meses de vida ${ }^{10}$.

\section{Biomarcadores}

El péptido natriurético tipo $\mathrm{B}$ (BNP) es un péptido hormonal secretado por los ventrículos cardíacos en respuesta a la tensión de pared ventricular. El uso de $\mathrm{BNP}$ o de su prohormona, el N-terminal pro BNP(NTproBNP) ha ido en aumento para el seguimiento de pacientes con HP asociado a DBP. Sin embargo, ocasionalmente se encuentran RN con elevación de BNP cuyos ecocardiogramas no muestran elevación significativa de la presión de ventrículo derecho, y otros en que el BNP es bajo a pesar de que el ecocardiograma muestra signos de HP.

En estudios recientes, (Kalra VK et al 2014, Sellmer et al 2015), se encontró una asociación significativa de la elevación de BNP plasmático con DBP grave y de
NT-proBNP con DBP o mortalidad, sugiriendo que la medición de estos en neonatos con DBP podría ayudar a estratificación de riesgo e implementación de otras terapias dirigidas $^{13,14}$.

Dado que es un examen poco específico al elevarse también en otras patologías que producen aumento en la tensión de pared ventricular como hipertensión sistémica, ductus arterioso persistente y disfunción ventricular izquierda; además de existir valores de cortes poco claros, la medición de biomarcadores no constituiría una herramienta diagnóstica, pero podría ser útil para el seguimiento de los pacientes y eventualmente para evaluar respuesta a terapias específicas.

\section{Prevención y Tratamiento}

A pesar de la importante morbimortalidad asociada a la DBP con HP, no existen en la literatura estudios controlados aleatorizados que avalen las diversas tera- 
pias que están en uso actualmente. El actuar médico se ha basado en estudios observacionales, reportes de caso y algunos CRT únicamente enfocados en la disminución en DBP, y ha sido la experiencia junto a éstos las principales herramientas que guían las decisiones a tomar con estos pacientes. Es bajo este marco en que se deben interpretar los resultados descritos y las sugerencias de terapias, esperando en un futuro avanzar en este campo de investigación.

\section{Prevención con iNO}

Durante los últimos años ha habido creciente interés en el potencial rol del Óxido Nítrico Inhalado (iNO) como terapia de prevención de la DBP con HP. Esto en el contexto de su base fisiopatológica -asociación entre defectos en la "señalización" del NO y remodelación pulmonar aberrante- y por diversos estudios en modelos animales con resultados promisorios. En éstos, se obtuvo disminución de la inflamación pulmonar, del stress oxidativo y promoción de la alveolarización y crecimiento pulmonar. Sin embargo, los estudios controlados aleatorizados reunidos en un metaanálisis Cochrane reciente, con más de 3000 prematuros enrolados en más de 12 estudios, no lograron demostrar disminución del riesgo de displasia broncopulmonar. Actualmente, las indicaciones para iNO son más bien terapéuticas y reservadas para crisis de hipertensión pulmonar en dosis de 5-20 ppm en pacientes seleccionados, siguiendo así lo propuesto en el consenso del NIH 2011 16,17,18. En la búsqueda de otras opciones para intervenir la vía alterada de NO y poder aumentar su disponibilidad, han aparecido estudios respecto a la suplementación con L-arginina y L-citrulina, siendo esta segunda la más prometedora ya que se han reportado efectos adversos en los ensayos clínicos con L-arginina. Aún faltaría traducir los resultados alentadores encontrados en animales a estudios en humanos ${ }^{19}$.

\section{Soporte ventilatorio y oxígenoterapia}

Esta bien descrito el rol del oxígeno como vasodilatador y por ello es por lo que una de las estrategias de prevención de HP en RN con DBP es mantener una ventilación gentil con objetivos de saturación 91-95\% para evitar la vasoconstricción y el desarrollo de enfermedad vascular pulmonar en los pacientes de riesgo. En los casos en que el paciente desarrolla DBP con HP es esencial impedir períodos de hipoxemia crónicos o episódicos, para lo cual es necesario realizar estudios de saturometría y hasta eventualmente polisomnografía y/o capnografía en algunos casos específicos ${ }^{20}$.

\section{Terapias complementarias}

En estos pacientes, tanto en el estudio como en el manejo se debe partir por el tratamiento de la enferme- dad pulmonar subyacente. Esto incluye evaluar y tratar patologías asociadas como el reflujo gastroesofágico crónico y aspiración, alteraciones estructurales de la vía aérea, hiperreactividad bronquial, congestión pulmonar etc. Junto a esto, el manejo nutricional de estos pacientes constituye un pilar fundamental, ya que para el crecimiento y desarrollo adecuado del aparato respiratorio es necesaria una nutrición adecuada según los requerimientos del individuo, ajustándose a los cambios que éste presente y manteniéndome un estricto seguimiento de sus curvas de crecimiento. A su vez, es importante tomar en consideración que, sin el soporte ventilatorio adecuado, el mayor esfuerzo respiratorio se asocia a mal incremento ponderal ${ }^{20,21}$.

\section{Terapias específicas}

La búsqueda de agentes de prevención o tratamientos para la $\mathrm{HP}$ asociada o no a DBP, han dado pie a un gran número de estudios para evaluar diferentes agentes como, los inhibidores de fosfodiesterasa $(3 \mathrm{y}$ 5), los análogos de prostaciclinas y los antagonistas del receptor de endotelina. El agente más ampliamente usado como monoterapia, con relativa seguridad y eficiencia es el sildenafil (inhibidor de fosfodiesterasa 5), el que se recomienda iniciar en dosis de $0,5 \mathrm{mg} /$ $\mathrm{kg}$ cada 8 horas para luego aumentar hasta un máximo de $2 \mathrm{mg} / \mathrm{kg}$ cada 6 horas. Su principal efecto es la reducción de la resistencia vascular pulmonar y por ende la presión arterial pulmonar en forma preferente, pero con un potencial efecto sistémico. Debido a esto último, se debe vigilar la aparición de hipotensión sistémica como efecto adverso a su uso. Con esta droga sólo existen series retrospectivas de menos de 25 pacientes cada una las que en grado variable han mostrado mejoría ecocardiográfica y clínica de su $\mathrm{HP}^{22,23,24,25}$. El estudio más completo parece ser el de Trottier-Boucher et al, que muestra una mejoría ecocardiográfica y clínica en 74 y $35 \%$ de sus pacientes respectivamente. El mismo estudio, reporta un $44 \%$ de pacientes que presentaron hipotensión sistémica asociada al medicamento ${ }^{22}$. En el mismo sentido, si uno evalúa la sobrevida de las series reportadas de pacientes que han recibido terapia con sildenafil, todas ellas muestran una sobrevida (entre 66 a 95\%) que es mayor a la reportada en las series con HP y DBP que no han recibido tratamiento. Lo anterior, obviamente, no es equivalente a un estudio controlado, pero es la evidencia disponible hoy en día, que nos hace pensar que el Sildenafil si es una alternativa terapéutica efectiva. Otro agente evaluado es el bosentán, antagonista de los receptores de endotelina, ETa y ETb, que ha mostrado ser bien tolerado y aportar a la mejoría clínica en pacientes pediátricos con $\mathrm{HP}^{26}$. En pacientes displásicos con HP, sólo una serie de Kadmon et al ha sido publicada donde fue usado asociado a sildenafil 
en 5 niños y como monoterapia en 1, con buenos resultados tanto clínicos como ecográficos ${ }^{27}$. En cuanto a los análogos de prostaciclinas, como el iloprost, es poca la evidencia disponible sobre su uso en menores de 2 años. Sin embargo, Piastra et al el 2012 da cuenta de una serie de 10 pacientes que recibieron iloprost inhalado con buenos resultados, pero por un tiempo acotado. Es difícil imaginar una terapia como ésta mantenida en el tiempo por lo engorroso que resulta su administración y relativamente frecuentes broncoespasmos asociados a su administración. Prostanoides de uso sistémico como epoporostenol han sido usado en algunas series, pero causan hipotensión sistémica lo que limita su uso. Con la evidencia disponible en niños, las guías clínicas de la AHA para la HP del 2015 concluyen que los usos de estas terapias específicas pueden ser útiles cuando se han manejado todas las causas subyacentes tanto pulmonares como cardía$\mathrm{cas}^{12}$. En nuestro centro empleamos habitualmente inicio de tratamiento con sildenafil como primera línea cuando los pacientes presentan HP secundaria a DBP severa, luego de haber evaluado y tratado pertinentemente otras posibles etiologías subyacentes. En caso de no responder, se podría agregar bosentán o iloprost según la experiencia del equipo tratante y las características del paciente específico ${ }^{8,21}$.

\section{Retos futuros}

El mayor desafío a futuro es encontrar terapias preventivas efectivas de DBP, y por ende de sus complicaciones. Dada la clara asociación de mayor mortalidad y morbilidad en pacientes con DBP que presentan $\mathrm{HP}$, queda pendiente aún desarrollar un protocolo de screening para diagnosticar tempranamente a estos pacientes, realizar una estratificación de riesgo e iniciar tratamiento. La ecocardiografía transtorácica, al ser un método no invasivo, actualmente es la primera alternativa de evaluación de pacientes con DBP e HP asociada, por lo que la realización de ecocardiografía seriada podría ser una buena alternativa para el diagnóstico y seguimiento de estos pacientes, faltando mayores estudios que definan el momento más apropiado para realizarse. Actualmente no existe evidencia suficiente en cuanto a estrategias preventivas de esta asociación ni de manejo de estos pacientes, pero pareciera adecuado mantener una saturación de oxígeno 91-95\%, evitando eventos hipoxémicos, así como también el uso de vasodilatadores pulmonares como, sildenafil en casos más graves. Sin embargo, aún se requiere de mayor investigación en cuanto al fenotipo específico de DBP con HP y de esta forma poder realizar una estrategia de prevención y terapia dirigida.

\section{Referencias}

1. Northway W, Rosan R, Porter D. Pulmonary Disease Following Respirator Therapy of Hyaline-Membrane Disease. N Engl J Med 1967; 276(7):357-68.

2. Kinsella JP, Greenough A, Abman SH. Bronchopulmonary dysplasia. Lancet 2006;367(9520):1421-31.

3. Stoll BJ, Hansen NI, Bell EF, et al. Neonatal outcomes of extremely preterm infants from the NICHD Neonatal Research Network. Pediatrics 2010;126(3):443-56.

4. Tapia JL, Agost D, Alegria A, Standen J, Escobar M, Grandi C, et al. Bronchopulmonary dysplasia: incidence, risk factors and resource utilization in a very low birth weight infant Southamerican population. J Pediatr (Rio J) 2006; 82:15-20.

5. Khemani E, McElhinney DB, Rhein L, et al. Pulmonary Artery Hypertension in Formerly Premature Infants with Bronchopulmonary Dysplasia: Clinical Features and Outcomes in the Surfactant Era. Pediatrics 2007;120(6):1260-9.

6. Al-Ghanem G, Shah P, Thomas S, Banfield L, el Helou S, Fusch C, et al. Bronchopulmonary dysplasia and pulmonary hypertension: a meta-analysis.
J Perinatol 2017;37(4):414-9.

7. Mourani P, Abman S. Pulmonary Hypertension and Vascular Abnormalities in Bronchopulmonary Dysplasia. Clin Perinatol 2015;42(4):839-55.

8. Ambalavanan N, Mourani P. Pulmonary hypertension in bronchopulmonary dysplasia. Birth Defects Part A: Birth Defects Res A Clin Mol Teratol 2014;100(3):240-6.

9. Carlton E, Sontag M, Younoszai A, DiMaria M, Miller J, Poindexter B et al. Reliability of Echocardiographic Indicators of Pulmonary Vascular Disease in Preterm Infants at Risk for Bronchopulmonary Dysplasia. J Pediatr 2017; 186:29-33.

10. del Cerro M, Sabaté Rotés A, Cartón A, Deiros L, Bret M, Cordeiro M et al. Pulmonary hypertension in bronchopulmonary dysplasia: Clinical findings, cardiovascular anomalies and outcomes. Pediatr Pulmonol 2014;49(1):49-59.

11. Mourani P, Sontag M, Younoszai A, Ivy D, Abman S. Clinical Utility of Echocardiography for the Diagnosis and Management of Pulmonary Vascular Disease in Young Children with Chronic Lung Disease. Pediatrics 2008;121(2):31725 .
12. Abman SH, Hansmann G, Archer SL, et al. Pediatric Pulmonary Hypertension: Guidelines from the American Heart Association and American Thoracic Society. Circulation. November 2015. doi:10.1161/CIR.0000000000000329.

13. Kalra VK, Aggarwal S, Arora P, Natarajan G. B-Type Natriuretic Peptide Levels in Preterm Neonates with Bronchopulmonary Dysplasia: A Marker of Severity? Pediatr Pulmonol 2014. 49:1106-11.

14. Sellmer A, Hjortdal V, Bjerre J, Schmidt M, McNamara P, Bech $B$ et al. N-Terminal Pro-B Type Natriuretic Peptide as a Marker of Bronchopulmonary Dysplasia or Death in Very Preterm Neonates: A Cohort Study. PLOS ONE. 2015;10(10): e0140079.

15. Barrington KJ, Finer NN, Pennaforte T. Inhaled nitric oxide for respiratory failure in preterm infants. Cochrane Database Syst Rev. 2017;1:CD000509. doi:10.1002/14651858.CD000509.pub5.

16. Arul N, Konduri G. Inhaled Nitric Oxide for Preterm Neonates. Clin Perinatol 2009; 36(1):43-61.

17. Raffay T, Martin R, Reynolds J. Can Nitric Oxide-Based Therapy Prevent Bronchopulmonary Dysplasia? Clin Perinatol 2012;39(3):613-38. 
18. Cole F, Alleyne C, Barks J, Boyle R, Carroll J, Dokken D, et al. NIH Consensus Development Conference Statement: Inhaled Nitric-Oxide Therapy for Premature Infants. Pediatrics 2011;127(2):363-9.

19. Fike C, Summar M, Ashner J. L-citrulline Provides a Novel Strategy for Treating Chronic Pulmonary Hypertension in Newborn Infants. Acta Paediatr 2014; 103:1019-26.

20. Abman SH, Collaco JM, Shepherd EG, Keszler M, Cuevas-Guaman M, Welty SE et al. Interdisciplinary Care of Children with Severe Bronchopulmonary Dysplasia. J Pediatr 2017; 181:12-28.

21. Wardle A, Wardle R, Luyt K, Tulloh R. The utility of sildenafil in pulmonary hypertension: a focus on bronchopulmonary dysplasia. Arch Dis Child 2013;98(8):613-7.

22. Trottier-Boucher MN, Lapointe A,
Malo J, et al. Sildenafil for the Treatment of Pulmonary Arterial Hypertension in Infants with Bronchopulmonary Dysplasia. Pediatr Cardiol 2015;36(6):1-6.

23. Tan K, Krishnamurthy MB, O’Heney JL, Paul E, Sehgal A. Sildenafil therapy in bronchopulmonary dysplasia-associated pulmonary hypertension: a retrospective study of efficacy and safety. Eur J Pediatr 2015;174(8):1109-15.

24. Nyp M, Sandritter T, Poppinga N, Simon C, Truog WE. Sildenafil citrate, bronchopulmonary dysplasia and disordered pulmonary gas exchange: any benefits \& quest. Nature Publishing Group. 2011;32(1):64-69. doi:10.1038/ jp.2011.131.

25. Mourani PM, Sontag MK, Ivy DD, Abman SH. Effects of long-term sildenafil treatment for pulmonary hypertension in infants with chronic lung disease. J Pediatr 2009;154(3):379-84.
26. Rosenzweig EB, Ivy DD, Widlitz A, et al. Effects of Long-Term Bosentan in Children with Pulmonary Arterial Hypertension. J Am Coll Cardiol. 2005;46(4):697-704.

27. Kadmon G, Schiller O, Dagan T, Bruckheimer E, Birk E, Schonfeld T. Pulmonary hypertension specific treatment in infants with bronchopulmonary dysplasia. Pediatr Pulmonol 2016;52(1):77-83.

28. Krishnan Usah, Rosenzweig E. Pulmonary Hypertension in Chronic Lung Disease of Infancy. Current Opinion in Pediatrics. 2015;27(2):177-84.

29. Berkelhamer SK, Mestan KK, Steinhorn RH. Pulmonary Hypertension in Bronchopulmonary Dysplasia. Sem Perinatol 2013;37(2):124-31.

30. Steinhorn R. Diagnosis and Treatment of Pulmonary Hypertension in Infancy. Early Hum Dev 2013; 89:865-74. 